\title{
An asymmetrical fenestration of the basilar artery coexisting with two aneurysms in a patient with subarachnoid haemorrhage: case report and review of the literature
}

\author{
M. Polguj', M. Majos¹, 2, M. Topol ${ }^{3}$ A. Majos² \\ ${ }^{1}$ Department of Angiology, Chair of Anatomy, Medical University of Lodz, Poland \\ 2Department of Radiology, Medical University of Lodz, Poland \\ ${ }^{3}$ Department of Normal and Clinical Anatomy, Chair of Anatomy, Medical University of Lodz, Poland
}

[Received 3 September 2013; Accepted 25 October 2013]

\begin{abstract}
The vertebrobasilar system is a part of the cerebral arterial circle (circle of Willis), which forms the collateral circulation of the brain. A 75-year-old Caucasian female was admitted to hospital because of a strong headache radiating to the neck. On the basis of a neurological examination, the patient was classified into group III of the Hunt and Hess scale. Subarachnoid haemorrhage and 2 aneurysms of the cerebral arteries were diagnosed during multidetector 64-row computed tomography and angiography. An asymmetrical fenestration of the proximal part of the basilar artery was also observed. The bleeding aneurysm locating at anterior communicating artery was diagnosed and clipped surgically by right fronto-parietal craniotomy. The second aneurysm was located just after the junction of the vertebral arteries on the wall of the basilar artery. The presented case firstly illustrates the asymmetric fenestration of the proximal part of the basilar artery coexisting with subarachnoid haemorrhage and 2 aneurysms of brain arteries. Such observation should increase diagnostic attention in the detection of possible associated aneurysms and can help in preventing complications during all endovascular treatment procedures. (Folia Morphol 2014; 73, 2: 229-233)
\end{abstract}

Key words: basilar artery, fenestration, computed tomography angiography, variations, aneurysm

\begin{abstract}
INTRODUCTION
The vertebrobasilar system (VBS) is formed by the intracranial parts of the vertebral arteries (VAs), the basilar artery (BA) and its branches. The VBS supplies several important structures, such as the spinal cord, brain stem and cerebellum, latero-medial aspects of occipital lobe, as well as the inferior aspect of the temporal lobe of the brain. It also comprises a part of the cerebral arterial circle (circle of Willis), which forms the collateral circulation of the brain [15].
\end{abstract}

Identification of variations in the VBS is clinically important because they should be taken into consideration when catherisation is needed. It has been also speculated that some anomaly of the arteries of the VBS (e.g. duplication or fenestration) may predispose them more to the dissection [18].

The presented case firstly illustrates the asymmetric fenestration of the proximal part of the BA. This anomaly was recognised in a patient with subarachnoid haemorrhage $(\mathrm{SAH})$ and 2 aneurysms of brain 


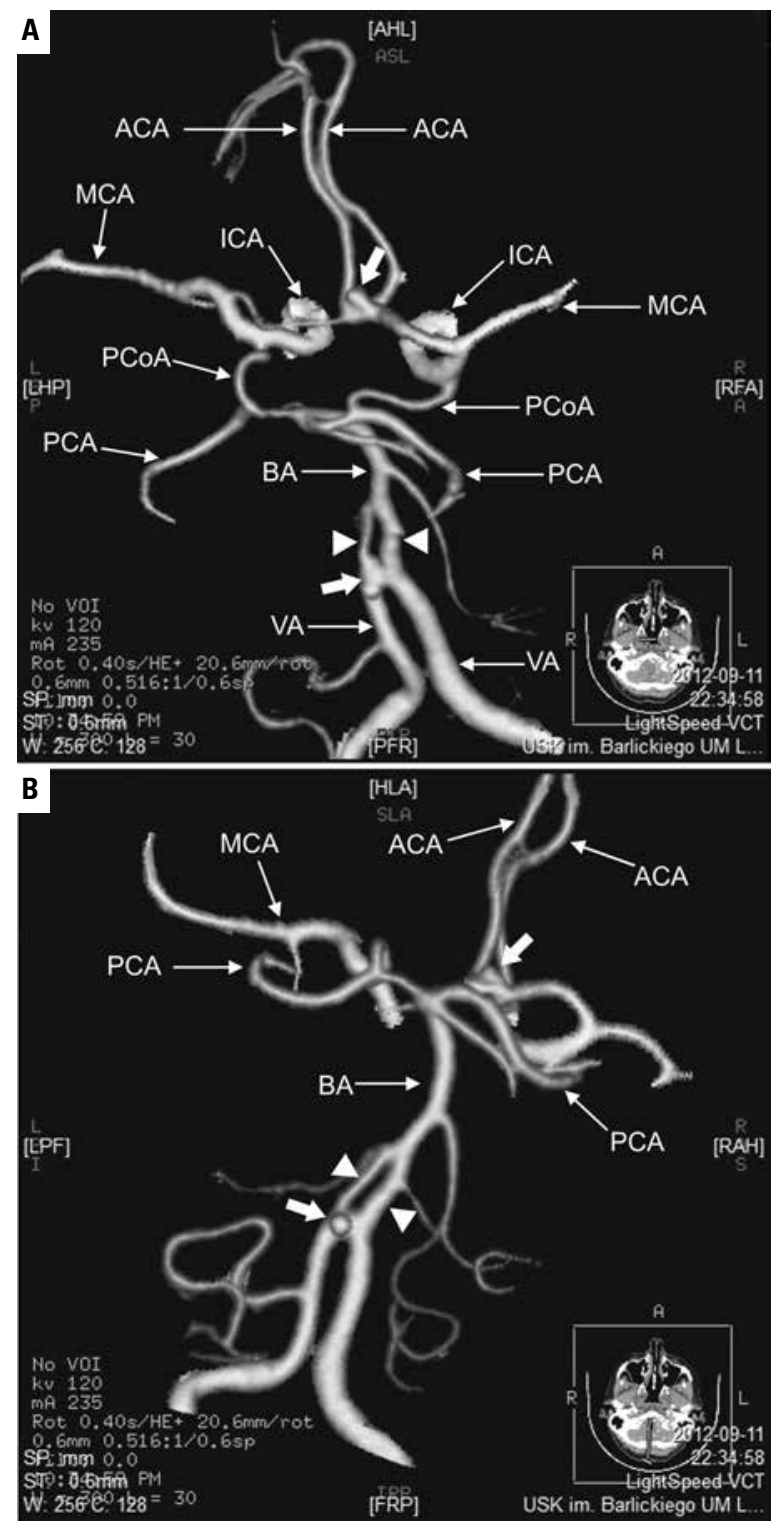

Figure 1A, B. Three-dimensional computed tomography reconstruction of the arteries of head. Arrows - aneurysms; arrowhead fenestrated basilar artery; ACA - anterior cerebral artery; $\mathrm{BA}$ — basilar artery; ICA — internal carotid artery; MCA — middle cerebral artery; PCA — posterior cerebral artery; PCoA — posterior communication artery; $\mathrm{VA}$ - vertebral artery.

arteries. Such observation should increase diagnostic attention in detection of possible associated aneurysms and can help in preventing complications during endovascular treatment.

\section{CASE REPORT}

A 75-year-old Caucasian female was admitted to the neurological department of our hospital for SAH. On the day of arrival she was suffering from a strong headache radiating to the neck, which

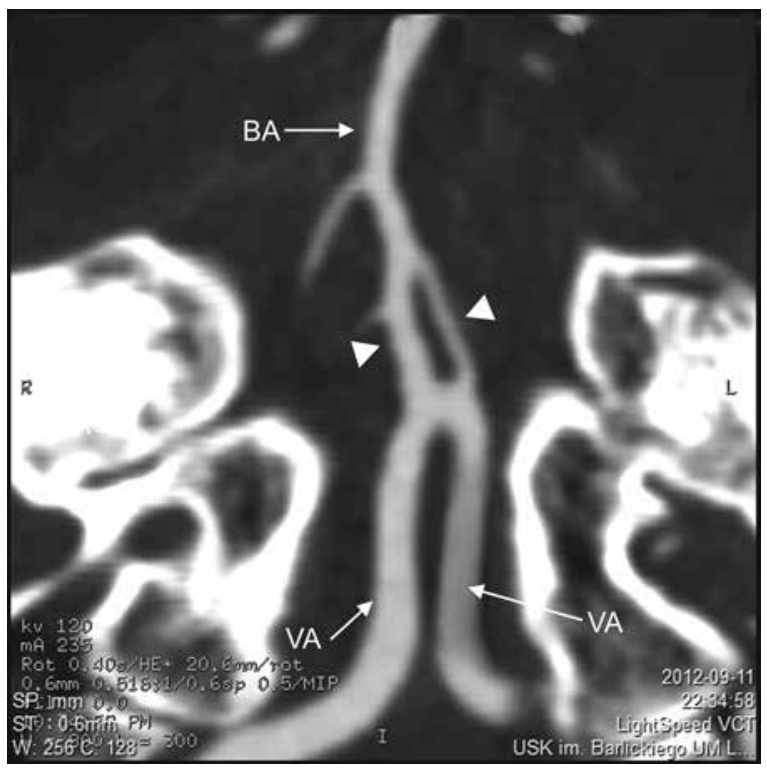

Figure 2. Helical computed tomography angiography, multiplanar reconstruction in transverse plane of the head: arrowhead - fenestrated basilar artery; BA — basilar artery; VA — vertebral artery.

was also a reason of a single episode of loss of the consciousness. On admission, she was conscious, but dozing. On the basis of the neurological examination, she was classified into group III of the Hunt and Hess scale. She has never smoked and never controlled her blood pressure. Her family medical history revealed that a sister had experienced several brain strokes.

Two aneurysms were reported in the angio-computed tomography examination (64-row MDCT scanner, LightSpeed VCT, GE, Waukesha, Wisconsin, USA) performed on the day of admission; one was a $3 \times 3 \mathrm{~mm}$ aneurysm of the anterior communicating artery (ACOA), while the second, measuring $3 \times 4 \mathrm{~mm}$, was located just after the junction of the VAs on the wall of the BA (Fig. 1). What is more, a $12 \mathrm{~mm}$ fenestration of the proximal part of BA was also observed (Figs. 1, 2). The fenestrated parts had different transverse diameters: the left branch measured $1.9 \mathrm{~mm}$, the right one $-3.0 \mathrm{~mm}$. The diameters of the VAs in the same plane were $2.5 \mathrm{~mm}$ for the left, and $3.0 \mathrm{~mm}$ for the right. The width of the BA above the fenestration was the same as just before the division into the posterior cerebral arteries: $2.8 \mathrm{~mm}$ (Fig. 3).

The cerebral angiography (Axion Artis, Simens, Germany) performed from a right femoral artery puncture confirmed the presence of the aneurysm of the ACoA, as well as the fenestration of the BA (Fig. 4). 


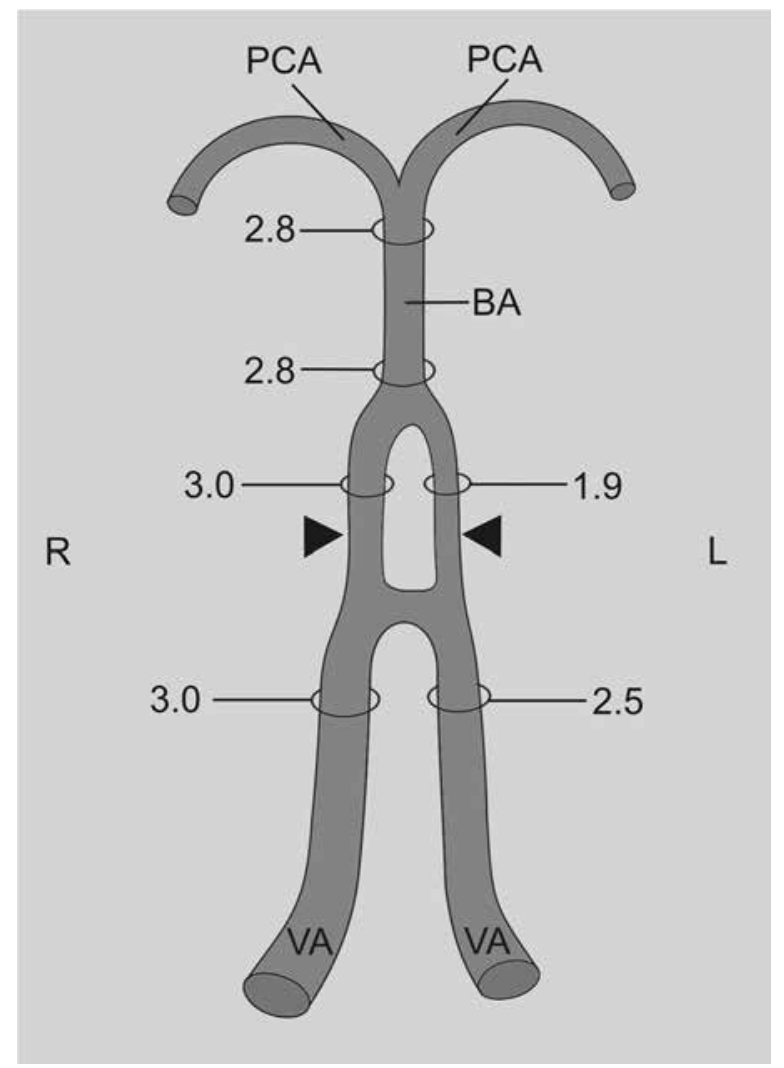

Figure 3. Schematic arrangements of the vessels of vertebrobasilar system and measurements of diameter of the arteries [mm]: arrowhead — fenestrated basilar artery; BA — basilar artery; PCA — posterior cerebral artery; VA — vertebral artery.

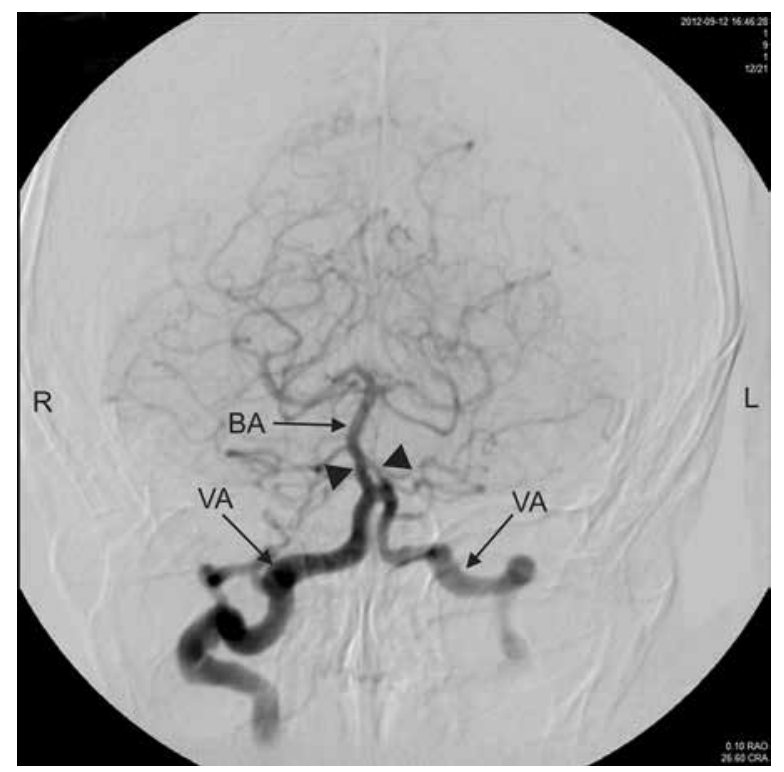

Figure 4. Angiography of the vessels of head: arrowhead fenestrated basilar artery; BA - basilar artery; VA - vertebral artery.
The second aneurysm was also verified with exactly the same location and size as observed in the angio-computed tomography examination. No complications were reported during or after the angiography.

The bleeding aneurysm locating at ACoA was diagnosed and clipped surgically by right fronto-parietal craniotomy. The operation ended with no neurological deficits, the patient obeyed the orders correctly.

\section{DISCUSSION}

The incidence of fenestration of the BA in an autopsy series is estimated between $4 \%$ and $6 \%[3,12]$. However, Songur et al. [20] described the fenestration of BA in the proximal segments only in $0.9 \%$ of cases and in distal segments in $1.8 \%$ of cases, based on their own anatomical studies. The frequency of fenestration of the BA observed in angiographic series is much rarer - in the range of $0.1-1.9 \%[1,15]$. The reason for the discrepancy between the frequencies of fenestration of the BA may be the population variability. Nevertheless, another reason could be that 2 different techniques were used: the first being an anatomical study, and the second a radiological visualisation. It is supported by Okahara et al. [15], who confirm that, as opposed to anatomical studies, small fenestrations are not visible in conventional digital subtraction angiography (DSA), and are only detectable by 3-dimensional reconstruction images from rotational DSA.

A pathomorphological study by Black and Ansbacher [4] describes defects in the vessel wall of each end of fenestration, which may promote the growth of the aneurysm due to haemodynamic forces and structural degenerative changes in the vessel. Also, Finlay and Canham [8] note that in some parts of the fenestration, there is no tunica media, and the elastin filaments were discontinuous at the proximal and distal ends of the fenestration. In Tsuei et al. [22] opinion, the histological and the haemodynamic factors to which the arterial wall of the proximal portion of the fenestration is directly exposed, such as the shearing stress from the blood pressure, also play an indispensable role in the formation of the aneurysm. Also, Yoon et al. [24] suppose that blood flow to the fenestration limb may cause haemodynamic stress to the vessel wall, inducing aneurysm formation. In Berry et al. [3] opinion, haemodynamic disturbances and turbulence, caused by endothelium-lined partial intraluminal septa, make the possibility of a thrombus or embolus formation much higher in a fenestrated 
artery than in a normal artery. Also, Kloska et al. [14] reported a case of a brainstem infarction in a child with BA fenestration. In their opinion, paediatric arterial thromboembolic stroke may have been caused by 2 abnormalities, including BA fenestration and a coagulation disorder.

Tasker and Byrne [21] have suggested that aneurysms associated with fenestration tend to have broader necks than those of a comparable size at other sites, and may therefore be more difficult to treat successfully via the endovascular route. Also, Ezaki et al. [6] stated that because of the risk of coil deposit in the parent artery, the complete occlusion is less probable in the wide-necked aneurysms. However, in the aneurysms associated with fenestration, even when 1 limb is occluded by coiling, cerebral blood flow may be preserved if the other limb of the fenestration is patent. Islak et al. [13] and Yoon et al. [24] described the cases in which one of the fenestration limb was occluded during the endovascular procedure. No neurologic deterioration occurred in their patients. It was confirmed by Peluso at al. studies [17]. Scientists presented results of endovascular treatment with coil occlusion of the 7 vertebrobasilar junction aneurysms associated with fenestration. All aneurysms were adequately occluded without vascular complications. Also, Islak et al. [13] described that 10 of 11 basilar artery fenestration aneurysms were occluded completely. They reported that of the 11 cases, 5 were cases of wide-necked aneurysms, and the balloon remodelling technique was used in 3 cases [19].

In contrary, Fujimoto et al. [9] stated that surgery of basilar fenestration aneurysm may be difficult because of the complex geometry of the fenestration, difficulty in obtaining adequate surgical exposure, and crowding of arteries in this region. It is supported by Campos et al. report [5]. In their study, basilar artery fenestration aneurysms were surgically treated and 17 aneurysms could be clipped, with total occlusion in $14(70 \%)$ cases and incomplete clipping in $3(15 \%)$ cases. In $13(65 \%)$ cases, the patients had post-operative transient lower cranial nerve palsies; 1 had permanent severe neurologic deficit and 1 patient died.

Surgical access to the vertebrobasilar junction region is difficult, and local anatomy is complex due to the presence of lower cranial nerves and perforators to the brain stem. Therefore, endovascular treatment of aneurysms in this region with detachable coils is now widely accepted $[7,17,24]$.
However, complicated geometry of the fenestration sometimes makes the clipping of the vertebrobasilar junction aneurysms difficult. Gruber et al. [10] described the case of fenestration of BA including tortuous shape of 1 limb coexisting with aneurysm. This anatomic configuration would make stenting of these segments more difficult and possibly produce kinking of the stent.

In our study, the diameter of the basilar artery was $2.8 \mathrm{~mm}$, which was smaller than normal [12, 20]. Idowu et al. [12] described the diameter of this vessel as $3.82 \pm 0.63 \mathrm{~mm}$, while Songur et al. [20] reported it to be $3.97 \pm 0.96 \mathrm{~mm}$. Such observation is important, because Fellgiebel et al. [7] reported that in patients with stroke, the diameter of BA was significantly lower $-2.45 \pm 0.5 \mathrm{~mm}$.

The BA is developed by coexistence of 2 processes: longitudinal and axial, going on in a $5^{\text {th }}$ prenatal week, when human embryo has 3-4 mm crown-rump length $[16,23]$. The longitudinal system follows the growth pattern found in anatomy of the spinal cord; the part of the BA is formed by a fusion of 2 ventral, longitudinal neural arteries [2]. In the same time, the axial system is developed by a union of caudal division of internal carotid artery in the ponto-mesencefalic sulcus [11]. These processes occur with the late involution of the trigeminal artery, which is responsible for flow changes in this anatomical region. Usually the BA is fully completed when embryo has 33 days and $9 \mathrm{~mm}$ crown-rump length $[16,23]$. In the embryonic period, several developmental anomalies of the cerebral arteries can occur. According to Padget [16], fenestration of the basilar artery is the result of a failure of the embryonic longitudinal neural arteries to fuse in the $5^{\text {th }}$ gestational week.

Knowledge of the topography and variations of the vertebrobasilar system is useful for diagnostic radiologist and endovascular interventionist. This has become more important in the era of new therapeutic options for intracranial intervention including use of vertebral artery stents, endovascular thrombectomy and endovascular thrombolysis $[17,19,24]$. To the best of our knowledge, asymmetrical fenestration of the basilar artery together with coexistence of the SAH and 2 aneurysms has not been previously reported. The lumen of 1 part of the fenestrated BA was found to be smaller than normal. In this event, interventional procedures focused on the middle or distal part of the BA should be performed from the higher part, if possible. 


\section{ACKNOWLEDGEMENTS}

The paper was supported by grant No. 503/2-031-01/ /503-01 from the Medical University of Lodz, Poland.

\section{REFERENCES}

1. Albanese E, Russo A, Ulm AJ (2009) Fenestrated vertebrobasilar junction aneurysm: diagnostic and therapeutic considerations. J Neurosurg, 110: 525-529.

2. Behari S, Krishna H, Kumar MV, Sawlani V, Phadke RV, Jain VK (2004) Association between an aplastic basilar artery, unaccompanied by a primitive carotid-vertebrobasilar anastomosis, and multiple aneurysms on the dominant posterior communicating artery. J Neurosurg, 100: 946-949.

3. Berry AD, Kepes JJ, Wetzel NM (1988) Segmental duplication of the basilar artery with thrombosis. Stroke, 119: 256-260.

4. Black SPW, Ansbacher LE (1984) Saccular aneurysm associated wit segmental duplication of the basilar artery. A morphological study. J Neurosurg, 61: 1005-1008.

5. Campos J, Fox AJ, Viñuela F, Lylyk P, Ferguson GG, Drake CG, Peerless SJ (1987) Saccular aneurysms in basilar artery fenestration. AJNR Am J Neuroradiol, 8: 233-236.

6. Ezaki Y, Kazekawa K, Tsutsumi K, Yagi N, Mori K, Kawakubo J, Shibayama A, Miyazaki H (2003) A vertebrobasilar junction aneurysm associated with fenestration treated by intra-aneurysmal embolization. Acta Neurochir, 145: 807-809.

7. Fellgiebel A, Keller I, Martus P, Ropele S, Yakushev I, Böttcher T, Fazekas F, Rolfs A (2011) Basilar artery diameter is a potential screening tool for Fabry disease in young stroke patients. Cerebrovasc Dis, 31: 294-299.

8. Finlay HM, Canham PB (1994) The layered fabric of cerebral artery fenestrations. Stroke, 25: 1799-1806.

9. Fujimoto K, Kawai S, Yonezawa T, Masui K, Nishi N, Maekawa M, Uranishi R (2007) Basilar trunk aneurysms with associated fenestration treated by using Guglielmi detachable coils: two cases reports. J Stroke Cerebrovasc Dis, 16: 84-87.

10. Gruber TJ, Ogilvy CS, Hauck EF, Levy El, Hopkins LN, Siddiqui AH (2010) Endovascular treatment of a large aneurysm arising from a basilar trunk fenestration using the waffle-cone technique. Neurosurgery, 67: 140-144.

11. Hoh BL, Rabinov JD, Pryor JC, Hirsch JA, Dooling EC, Ogilvy CS (2004) Persistent nonfused segments of the basilar artery: longitudinal versus axial nonfusion. Am J Neuroradiol, 25: 1194-1196.
12. Idowu OE, Malomo AO, Akang EU (2010) Surgical anatomy of the vertebrobasilar territory and posterior circle of Willis. West Afr J Med, 29: 230-234.

13. Islak C, Kocer N, Kantarci F, Saatci I, Uzma O, Canbaz B (2002) Endovascular management of basilar artery aneurysms associated with fenestrations. AJNR Am J Neuroradiol, 23: 958-964.

14. Kloska SP, Schlegel PM, Strater R, Niederstadt TU (2006) Causality of pediatric brainstem infarction and basilar artery fenestration? Pediatr Neurol, 35: 436-438.

15. Okahara M, Kiyosue H, Mori H, Tanoue S, Sainou M, Nagatomi $\mathrm{H}$ (2002) Anatomic variations of the cerebral arteries and their embryology: a pictorial review. Eur Radiol, 12: 2548-25461.

16. Padget DH (1948) The development of cranial arteries in thehumanembryo. Contrib Embryol, 32: 207-261.

17. Peluso JP, van Rooij WJ, Sluzewski M, Beute GN (2007) Aneurysms of the vertebrobasilar junction: incidence, clinical presentation, and outcome of endovascular treatment. Am J Neuroradiol, 28: 1747-1751.

18. Polguj M, Jędrzejewski K, Topol M, Wieczorek-Pastusiak J, Majos A (2013) Duplication of the left vertebral artery in a patient with dissection of the right internal carotid artery and Ehlers-Danlos syndrome - case report and review of the literature. Anat Sci Int, 88: 109-114.

19. Roth C, Mielke A, Siekmann R, Ferbert A (2011) First experiences with a new device for mechanical thrombectomy in acute basilar artery occlusion. Cerebrovasc Dis, 32: 28-34.

20. Songur A, Gonul Y, Ozen OA, Kucuker H, Uzun I, Bas O, Toktas M (2008) Variations in the intracranial vertebrobasilar system. Surg Radiol Anat, 30: 257-264.

21. Tasker AD, Byrne JV (1997) Basilar artery fenestration in association with aneurysms of the posterior cerebral circulation. Neuroradiology, 39: 185-189.

22. Tsuei YS, Matsumoto Y, Ohta M, Nakayama T, Ezura M, Takahashi A (2009) Vertebrobasilar junction fenestration with dumbbell-shaped aneurysms formation: computational fluid dynamics analysis. Surg Neurol, 72 (suppl. 2): S11-S19.

23. Vasović L, Trandafilović M, Jovanović I, Ugrenović S, Antović A, Karadžić R, Stojanović I. Human basilar artery abnormalities in the prenatal and postnatal period. World Neurosurg, 2013; 79: 593.e15-23.

24. Yoon SM, Chun YI, Kwon Y, Kwun BD (2004) Vertebrobasilar junction aneurysms associated with fenestration: experience of five cases treated with Guglielmi detachable coils. Surg Neurol, 61: 248-54. 